\title{
L'éducation des jeunes enfants au seuil de l'ère technologique
}

In: Communication et langages. N89, 3ème trimestre 1991. pp. 31-36.

\section{Résumé}

Le présent texte est issu du colloque organisé par l'Institut européen pour le développement des potentialités de tous les enfants (voir encadré ci-contre). Son auteur, professeur emérite de l'université de Liège, Gilbert de Landsheere, Prix international de l'Éducation, est l'auteur de travaux et de publications de grande notoriété sur les sciences de l'éducation et sur la lisibilité.

Citer ce document / Cite this document :

De Landsheere Gilbert. L'éducation des jeunes enfants au seuil de l'ère technologique. In: Communication et langages. $\mathrm{N}^{\circ} 89$, 3ème trimestre 1991. pp. 31-36.

doi : $10.3406 /$ colan.1991.2313

http://www.persee.fr/web/revues/home/prescript/article/colan_0336-1500_1991_num_89_1_2313 


\section{L'ÉDUCATION DES JEUNES ENFANTS AU SEUIL DE L'ÈRE TECHNOLOGIQUE}

par Gilbert de Landsheere

Le présent texte est issu du colloque organisé par l'Institut européen pour le développement des potentialités de tous les enfants (voir encadré ci-contre). Son auteur, professeur emérite de l'université de Llège, Gilbert de Landsheere, Prix International de l'Education, est l'auteur de travaux et de publications de grande notoriété sur les sciences de l'éducation et sur la lisibilité.

\section{LES TRANSACTIONS DE L'INVISIBLE}

Au moment où au moins tous les pays industrialisés entrent dans une ère nouvelle dont les bouleversements économiques, sociaux, culturels seront aussi importants que ceux de la Renaissance ou de la première révolution industrielle, l'Occident est-il aussi bien placé qu'il le devrait? Sa jeunesse - idealement toute sa jeunesse - est-elle prête à faire face aux problèmes qui l'attendent ? Rien n'est moins sûr. Pour y voir clair, il faudrait procéder d'urgence à une évaluation des besoins. Nous n'avons toutefois pas à attendre ses résultats pour savoir que notre société devient celle de l'intelligence, de l'information, de la complexité, du changement.

La libération des tâches routinières par l'informatique laisse une place prépondérante au jeu des processus mentaux supérieurs : analyse, synthèse, créativité. Dans une société qui, selon l'admirable expression de Louis Armand, est en train de s'encéphaliser, l'intelligence est devenue l'une des marchandises les plus recherchees : "Les échanges invisibles, écrit Caspar,' constituent désormais une part essentielle des échanges entre pays. Le Fonds monétaire international estime que la moitié de ces échanges se font desormais (...) sous forme de brevets, de hautes technologies, de capacités managériales,

1. Caspar P., La formation des adultes : besoin ou mythe?. Paris, Conservatoire national de arts et métiers, mai 1989, p. 2. 
d'informations financières ou stratégiques... bref, de savoirs. (...) Le savoir devient une variable stratégique. .

Société de l'information et de la communication. De la surinformation aussi, surinformation qui risque de déboussoler ou de paralyser ceux qui sont incapables de la trier, de la structurer. Société de la complexité. Société du changement accéléré. Le capital de connaissances de l'humanité doublerait, en moyenne, tous les sept ans. Les activités professionnelles - est-ce un hasard ? - demanderaient, elles aussi, une reconversion au même rythme. Changement encore dans tous les autres aspects sociaux.

Qui - individu, entreprise, nation - tire le mieux et le plus vite parti de ces conditions nouvelles est un gagneur. D'où la concurrence. Qui a le mieux compris ce qui arrive sait aussi que nous entrons dans l'ère de la mondialisation.

$\mathrm{Si}$, quittant l'échelle sociale, on s'interroge sur les caractéristiques individuelles requises, on s'aperçoit que l'individu total - intellectuel, affectif, moral, social, physique - est interpellé. Doué d'une bonne santé physique et mentale, ayant acquis dès le début, surtout dès le début de la scolarité, des bases solides, chacun doit idéalement pouvoir :

- prendre et analyser l'information et conquérir la connaissance :

- communiquer :

- être sensible à l'existence de problèmes et avoir l'envie et le pouvoir de les résoudre :

- élaborer des projets ;

- anticiper:

- travailler dans des conditions mouvantes, décider et agir sans posséder tous les éléments d'information souhaitables.

Est-il besoin de continuer pareil inventaire?

Croire que chacun possédera ces qualités à la perfection serait naîf. En revanche, considerer que nous trouvons là les caractéristiques modales souhaitées des populations de demain est tout simplement réaliste. II reste... à y arriver. Si nous le voulons vraiment, nous le pouvons. Comment ? En commençant par approfondir, sans complaisance aucune, lanalyse des problèmes majeurs d'éducation et de formation qui se posent. Des clignotants, annonciateurs de carences, se font de plus en plus vifs.

\section{DES EMPLOIS, MAIS FAUTE DE CAPACITÉS...}

Des clignotants

Ces avertisseurs de danger concernent d'abord l'instruction fondamentale, à commencer par les habiletés de base : comprehension de 
la lecture et des messages oraux, habileté à communiquer efficacement par la parole et par l'écrit, mathématiques, capacité de trouver l'information, acculturation informatique.

Méme si elles varient fortement en fonction de la définition de l'illettrisme adoptée, les statistiques sont alarmantes: à peu près certainement $10 \%$ de la population seraient illettrés en Belgique comme en France. Les résultats d'une étude française menée en 1986 ne sont pas moins inquiétants. À l'entrée dans l'enseignement secondaire supérieur, les bons lecteurs ne sont encore que $31 \%$. Or des données américaines indiquent que le niveau de difficulté de compréhension des textes que les travailleurs doivent lire dans les entreprises se situe, pour $70 \%$, au niveau moyen de l'enseignement secondaire supérieur, et pour $15 \%$, plus haut encore. Les carences en matière de compréhension des messages écrits sont d'autant plus dramatiques que la réalphabétisation des adultes est très difficile. La situation pour les autres habiletés intellectuelles de base n'est pas meilleure.

Les conséquences ne se sont pas fait attendre. Notre économie se porte bien. Mais il devient de plus en plus difficile de trouver des candidats minimalement compétents. Cela se comprend d'autant mieux que la moitié des emplois crés d'ici l'an 2000 exigeront une formation superieure, et environ un tiers seront destinés à des universitaires à part entière.

En 1988, l'ensemble des grandes entreprises des États-Unis ont dépensé ou perdu en productivité plus de 160 milliards de francs lourds pour faire acquérir les habiletés intellectuelles de base nécessaires à leur personnel. De grandes entreprises européennes annoncent aussi des carences graves dans ces domaines. Aux ÉtatsUnis encore, le budget consacré par les entreprises pour la formation supérieure de leur personnel dépasse l'ensemble des budgets des universités et des * collèges ^.

\section{FACE À L'ÉCHEC SCOLAIRE, QUE FAISONS-NOUS ?}

On sait le nombre élevé d'échecs scolaires, avoués ou non, dès les premières années de la scolarité, alors qu'elles sont les plus déterminantes. Certes, les chiffres souvent cités n'ont guère de sens. En réalité, il n'y a échec significatif que si des niveaux-cibles, opérationnellement définis, sont fixés au depart, si possible sous forme de contrat négocié, et que si l'on vérifie objectivement s'ils sont atteints ou non. Les informations sûres dont on dispose actuellement sont très parcellaires. On ne saura à quoi s'en tenir qu'à partir du moment où nos systèmes éducatifs seront systématiquement pilotés. 
Toutefois, les fondateurs de cet Institut ${ }^{2}$ vous expliqueront avec raison que la vraie solution n'est pas là. II faut, avant tout, empêcher que l'échec ne se produise et ne s'installe. II importe non seulement de prendre les mesures institutionnelles qui s'imposent, mais aussi de continuer la recherche.

Nous devons absolument rebatir comme il convient notre enseignement fondamental, c'est-à-dire bien sûr, le pré-scolaire et le primaire, mais aussi le secondaire. On continue souvent à y travailler avec des méthodes et des approches qui convenaient il y a 30-40 ans, à des populations sélectionnées sur le plan social et intellectuel, alors que les populations actuelles sont très hétérogènes, non seulement dans leurs aptitudes mais aussi dans leurs motivations, dans leurs buts.

Et l'on s'étonne que le système ne fonctionne pas ! $\mathrm{Si}$, aux prochains Jeux olympiques, on inscrivait toute la population française de 20 ans la moyenne baisserait assurément ... mais paradoxalement, il y aurait plus de champions! On parle toujours de la baisse de niveau, en oubliant de dire que les brillants n'ont jamais été aussi nombreux.

L'ensemble maternel-primaire-secondaire, qui constitue l'enseignement fondamental, a avant tout pour mission de diffuser une solide culture générale, elle aussi, reconstruite. La raison d'être de cette culture est d'offrir, aux futurs citoyens de l'an 2000, une nouvelle référence commune, une boussole culturelle et morale indispensable à ceux qui vont vivre dans le tourbillon des innovations techniques.

\section{UNE BOUSSOLE CULTURELLE}

Dans son recent ouvrage Que faut-il enseigner?, J.-M. Domenach ${ }^{3}$ cerne admirablement le rôle de la culture générale qui donne à tous un minimum de code commun. Il est grand temps, écrit en substance Domenach, d'opter de nouveau a pour des principes fermes, des méthodes éprouvées et un corpus qui recèle sa propre faculté d'adaptation, au lieu de chercher à rattraper les évolutions ». II faut donc rechercher l'essentiel dans le contemporain, le ramener à quelques règles, à quelques principes unifiants. Pour reprendre, une fois encore, la formule de Domenach, il importe * d'asseoir sur des bases simples et stables une pédagogie du complexe et du mobile $*$, tout en sachant bien * qu'entre la simplicité et la complexité, le chemin n'est pas univoque : on va de l'un à l'autre $\nsim$, et c'est dans ce va-etvient que se situe la dialectique de la pédagogie ( $p .35$ ).

Ce souhait d'un retour à une culture générale n'a rien d'accidentel.

2.Institut européen pour le développement des potentialités de tous les enfants (IEDPE).

3. Domenach J.-M., Que faut-il enseigner ? Paris, Le Seuil, 1989. 
Dans une situation où l'imprévisible, le changement, sont les constantes, des outils de reflexion alliant transcendance et fonctionnalité constituent les meilleures armes possibles. On n'insistera jamais assez sur l'urgence de reconstruire une boussole culturelle et morale à l'échelle de notre temps. Cette tâche est complexe et sa traduction en termes de programmes scolaires va demander au monde de l'éducation un énorme effort de réajustement.

Simultanément, toutes les disciplines d'enseignement, qu'il ne s'agit pas de fondre en un magma informe mais d'articuler, de coordonner, de " ponter ", doivent étre réexaminées pour décider de leur survie et de leur place, de leur esprit, de leur contenu. Cette remise en cause doit se faire en relation avec la réflexion relative à la culture générale nouvelle.

\section{LA FORMATION DES ENSEIGNANTS}

Un inventaire exhaustif des problèmes éducatifs actuels serait hors de propos ici. Il faut cependant en évoquer encore un parce qu'il est crucial : la qualité et la qualification des enseignants et des formateurs. Cette profession doit muter pour être à la hauteur des tâches qui lui sont dévolues et elle doit étre revalorisée en conséquence. Depuis des années, on improvise, on bricole, on esquive, on défend des droits acquis et des territoires. II est temps que cela cesse. On a bien admis que la restructuration de nos industries devait se faire quoi qu'il en coûte en argent et en problèmes humains. Pour l'avenir de nos nations, le problème de la formation de leurs enseignants et de leurs formateurs est plus important encore.

\section{SUR LA RECHERCHE EN ÉDUCATION}

Dans la majorité des États membres de la Communauté européenne. la recherche en éducation reste dans un état de sous-développement funeste, faute de ressources humaines et matérielles. Alors que, depuis longtemps, la cible minimale à atteindre par la recherche en éducation a été modestement fixée à $2 \%$ des budgets de l'éducation. il n'est pas exceptionnel que les dépenses effectives n'atteignent même pas $0,1 \%$.

Quelle entreprise, quelle armée survivraient dans pareilles conditions ? II n'est pas surprenant que la pédagogie demeure un domaine dans lequel toutes les affirmations gratuites restent possibles, et beaucoup, hélas!, fondent des décisions ou des actions dont les nations subissent les conséquences. Pour ses États membres, la Communauté européenne pourrait jouer un rôle décisif : on sait que son traité constituant l'en empêche. 
Comment les historiens de l'avenir pourront-ils vraiment comprendre que nos contemporains les plus éclairés, les plus conscients de la mutation culturelle que nous vivons, qui sont les premiers à proclamer que la recherche scientifique est la clé de l'avenir, que l'éducation est la priorité des priorités, ne tirent pas les conclusions qui s'imposent et n'agissent pas en conséquence?

Aussi longtemps que les ressources humaines et matérielles données à la recherche en education et en formation ne depasseront pas un seuil critique lui permettant de vraiment travailler à l'échelle indispensable et d'exercer une influence significative et systematique, nous continuerons à accumuler les pertes et à rater des occasions d'expansion et de progrès.

On peut beaucoup espérer de la Communauté européenne. Elle n'ira jamais assez vite pour faire entrer l'éducation officiellement dans ses responsabilités. II faut que, le plus vite possible, la Communauté européenne lance des programmes de recherche en éducation et en formation de la même importance que * Comet * ou * Esprit ». Notre avenir en dépend.

Gilbert de Landsheere 